\title{
Isolation of Candida dubliniensis in a French hospital mycology laboratory
}

Candida dubliniensis was first described as a novel species in 1995. The close phenotypic similarities between Candida albicans and C. dubliniensis have led to the misidentification of these species, which have to be distinguished genotypically (Sullivan et al., 2005). C. dubliniensis differs from $C$. albicans with respect to epidemiology, certain virulence characteristics and the ability to develop fluconazole resistance in vitro (Pinjon et al., 2005). The increasing number of reports of the recovery of C. dubliniensis from normal and human immunodeficiency virus (HIV)-infected patients suggests that it may be a constituent of the normal oral flora (Sullivan et al., 1997). Epidemiological studies have been performed elsewhere but, in France, information on the occurrence of $C$. dubliniensis versus $C$. albicans is still lacking.

The present investigation was a prospective study conducted over a 5 month period (from August to December 2005). During this period we studied all yeast isolates cultured on CHROMagar Candida medium (Becton Dikinson), a medium used routinely in our laboratory for fungal isolation from clinical samples. This medium allows selective isolation of yeasts, and simultaneously identifies C. albicans, Candida tropicalis and Candida krusei (Willinger \& Manafi, 1999). C. dubliniensis colonies usually have a darker green colour compared to C. albicans (Faggi et al., 2005). However, CHROMagar Candida does not clearly distinguish between $C$. albicans and C. dubliniensis (Mahnss et al., 2005), and therefore all isolates presumptively identified as C. albicans or C. dubliniensis were tested with a commercial latex agglutination test, Bichro-dubli test (Fumouze). The test consists of blue latex particles coated with the mAb 12F7-F2, which reacts specifically with an antigen on the surface of $C$. dubliniensis yeast cells. This agglutination slide test was validated on yeast strains previously identified by PCR, and had $100 \%$ sensitivity and specificity for $C$. dubliniensis isolated on either Sabouraud dextrose or CHROMagar Candida medium (Marot-Leblond et al., 2006). The anti-fungal susceptibility of the isolates of C. dubliniensis was determined using the Etest method according the recommendations of the manufacturer (AB Biodisk).

A total of 503 isolates (from 238 patients) were presumptively identified as either $C$. albicans or C. dubliniensis. Of these, 10 isolates from eight patients were identified with the Bichro-dubli test as $C$. dubliniensis. Among all Candida isolates identified in the laboratory during the survey, the ratio of $C$. dubliniensis to $C$. albicans was 10/493 (2.0\%). Interestingly, in seven of the ten samples in which $C$. dubliniensis was identified, it was associated with another Candida species, four times with Candida glabrata and on three occasions with C. albicans. As reported by others, we recovered $C$. dubliniensis predominately from HIVinfected patients. Six of our isolates were from four HIV-infected patients. These represented 4/24 (16.6\%) of all Candidapositive clinical samples from HIVinfected patients. Two of these were recovered from oral samples and four from the respiratory tract. Only 4 C. dubliniensis isolates were from non $\mathrm{HIV}$-infected patients versus 214 C. albicans, 4/214 $(1.8 \%)$, similar to the recovery rate reported for a Dutch university hospital (Meis et al., 2000). These four isolates came from: two burns patients (one from sputum and one from burned tissue), one patient in the Intensive Care Unit (sputum) and an out-patient with oral candidosis. Susceptibility testing showed that all the C. dubliniensis isolates had low MICs for: amphotericin B (0.012-0.032 $\mu \mathrm{g}$ $\left.\mathrm{ml}^{-1}\right)$, fluconazole $\left(0.094-0.125 \mu \mathrm{g} \mathrm{ml}^{-1}\right)$, voriconazole $\left(0.04-0.012 \mu \mathrm{g} \mathrm{ml}^{-1}\right)$ and caspofungin $\left(0.047-0.094 \mu \mathrm{g} \mathrm{ml}^{-1}\right)$. In the present study, 42 Candida isolates were obtained from the sputum of 32 cystic fibrosis patients. In contrast to a published report, in which a relatively high rate of $C$. dubliniensis colonization was reported $(11 \%)$ in this population (PeltrocheLlacsahuanga et al., 2002), we did not isolate any C. dubliniensis from our cystic fibrosis patients.

In conclusion, in a prospective study conducted in a hospital mycology laboratory in France, the proportion of $C$. dubliniensis/C. albicans isolated from non-HIV infected patient was low, 4/214 (1.8\%), as compared with $4 / 24(16.6 \%)$ from HIV-patients. C. dubliniensis was most often associated with another Candida species, and none of the 10 isolates of $C$. dubliniensis was resistant to fluconazole. These results emphasize the importance of using chromogenic medium in epidemiological studies and suggest preferential testing of presumptive $C$. albicans from HIV patients to distinguish $C$. dubliniensis.

\section{T. Baixench, C. Viguié and A. Paugam}

Laboratoire de Parasitologie-Mycologie, Hôpital Cochin, 75014 Paris, France

\section{Correspondence: A. Paugam} (andre.paugam@cch.aphp.fr)

\section{Faggi, E., Pini, G., Campisi, E., Martinelli, C. \& Difonzo, E. (2005). Detection of Candida dubliniensis in oropharyngeal samples from human immunodeficiency virus infected and non-infected patients and in a yeast culture collection. Mycoses 48, 211-215.}

Mahnss, B., Stehr, F., Schafer, W. \& Neuber, K. (2005). Comparison of standard phenotypic assays with a PCR method to discriminate Candida albicans and C. dubliniensis. Mycoses 48, 55-61.

Marot-Leblond, A., Beucher, B., David, S., Nail-Billaud, S. \& Robert, R. (2006).

Development and evaluation of a rapid latex agglutination test using a monoclonal antibody to identify Candida dubliniensis colonies. J Clin Microbiol 44, 138-142. 
Meis, J. F., Lunel, F. M., Verweij, P. E. \& Voss, A.

(2000). One-year prevalence of Candida

dublinienis in a Dutch university hospital. J Clin

Microbiol 38, 3139-3140.

Peltroche-Llacsahuanga, H., Dohmen, H. \&

Haase, G. (2002). Recovery of Candida

dubliniensis from sputum of cystic fibrosis

patients. Mycoses 45, 15-18
Pinjon, E., Moran, G. P., Coleman, D. C. \& Sullivan, D. J. (2005). Azole susceptibility and resistance in Candida dubliniensis. Biochem Soc Trans 33, 1210-1214.

Sullivan, D., Haynes, K., Bille, J., Boerlin, P., Rodero, L., Lloyd, S., Henman, M. \&

Coleman, D. (1997). Widespread geographic

distribution of oral Candida dubliniensis strains in human immunodeficiency virus-infected individuals. J Clin Microbiol 35, 960-964.

Sullivan, D. J., Moran, G. P. \& Coleman, D. C. (2005). Candida dubliniensis: ten years on. FEMS Microbiol Lett 253, 9-17.

Willinger, B. \& Manafi, M. (1999). Evaluation of CHROMagar Candida for rapid screening of clinical specimens for Candida species. Mycoses 42, 61-65. 